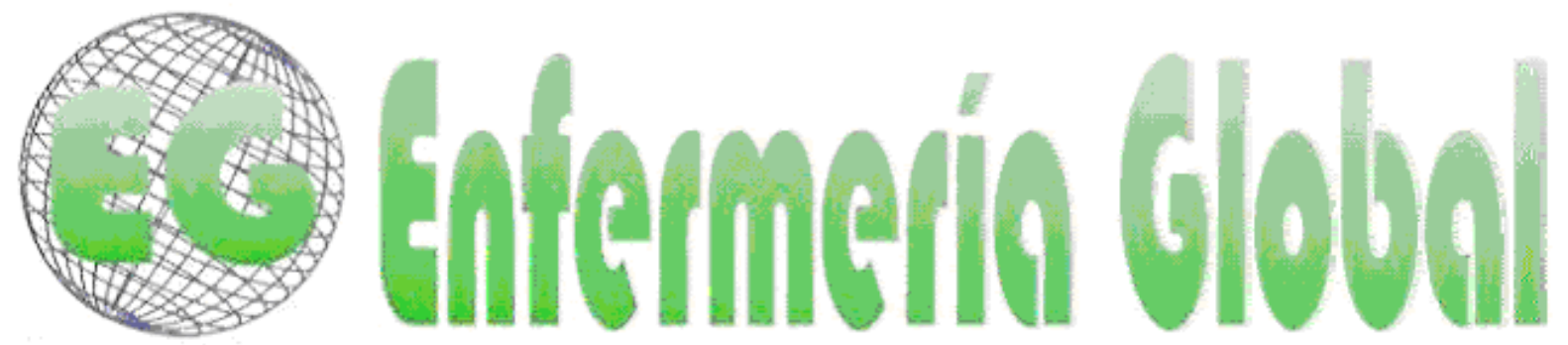

\title{
Ansiedad en mujeres con cáncer de mama
}

Ansiedade em mulheres com câncer de mama

\section{${ }^{*}$ Caniçali Primo, C. *Nunes Gonçalves, LR., **Pires Olympio, PC de A., ${ }^{* *}$ Costa Leite, FM., ${ }^{* * *}$ Costa Amorim, MH.}

\author{
*Enfermera graduada. E.mail:" candidaprimo@gmail.com **Máster en Salud Cominitaria. Profesora \\ del Curso de Graduación en Enfermería. ***Doctora en Enfermería. Profesora del Curso de \\ Graduación en Enfermería y del Programa de Pos-graduación en Enfermería e en Atención de Salud \\ Comunitaria. Universidad Federal do Espírito Santo - UFES - Vitória (ES), Brasil. \\ Palabras clave: neoplasias de la mama; ansiedad, mastectomia, enfermería. \\ Palavras-chave: neoplasias da mama; ansiedade; mastectomia; enfermagem.
}

Keywords: breast tumor; anxiety; mastectomy; nursing.

\section{RESUMEN}

Objetivo: Evaluar el indicio y el estado de ansiedad en mujeres mastectomizadas, y examinar la relación de la ansiedad con variables demográficas y clínicas.

Métodos: Se realizó un estudio descriptivo con 84 mujeres mastectomizadas, vinculadas al Programa de Rehabilitación para mujeres con mastectomía (Premma), ubicado en Vitória, Espírito Santo. Las variables se recogieron por medio de formulario específico, el indicio y estado de ansiedad tuvieron como base el Inventario de Ansiedad Indicio y Estado (IDATE).

Resultados: El trazo y el estado de ansiedad mostraron niveles promedio. Sólo el tiempo de tratamiento con que la mujer llegó a Premma estaba relacionado con la ansiedad $(p<0,05)$.

Conclusión: El que la mujer participe en un programa de rehabilitación interdisciplinar, tal vez, explique que los resultados no muestren niveles más altos de ansiedad.

\section{RESUMO}

Objetivo: Avaliar o traço e o estado de ansiedade em mulheres mastectomizadas e examinar a relação da ansiedade com variáveis sociodemográficas e clínicas.

Métodos: Estudo descritivo, realizado com 84 mulheres mastectomizadas vinculadas ao Programa de Reabilitação para Mulheres Mastectomizadas (PREMMA) localizado em Vitória/Espírito Santo. As 
variáveis foram coletadas por meio de formulário especifico; o traço e estado de ansiedade com base no Inventário de Ansiedade Traço e Estado (IDATE).

Resultados: $O$ traço e o estado de ansiedade apresentaram níveis médios. Apenas o momento do tratamento que a mulher chegou ao PREMMA apresentou relação com a ansiedade $(p<0,05)$.

Conclusão: A mulher participar de um programa de reabilitação interdisciplinar, talvez, justifique os escores não apresentarem níveis mais altos de ansiedade.

\section{ABSTRACT}

Objective: To evaluate traits and the state of anxiety in women who have had mastectomies and examine the relationship of anxiety with demographic and clinical variables.

Methods: A descriptive study was conducted with 84 women who have had mastectomies linked to the Rehabilitation Program for Women with Mastectomies (Premma) located in Vitória, EspíritoSanto. The variables were collected through specific form, the traits and state of anxiety on the basis of STAI- State Trait Anxiety Inventory.

Results: The traits and state of anxiety showed average levels. Only the time that the woman came to the treatment program was related to anxiety $(p<0.05)$.

Conclusion: Women participating in an interdisciplinary rehabilitation program, perhaps, justifies why the scores do not show high levels of anxiety.

\section{INTRODUCCIÓN}

El cáncer de mama es la neoplasia más común entre las mujeres y el segundo tipo de cáncer más frecuente en el mundo. Se estima que cada año, cerca de $22 \%$ de casos nuevos de cáncer en mujeres son de mama. En 2008, hubo en el país 10.208 óbitos por neoplasia de mama femenina. Para el año 2012, se estiman en Brasil 52.860 nuevos casos de cáncer de mama. El riesgo estimado para Brasil es de 52 casos por cada 100 mil mujeres; en la región Sudeste ese número sube a 68,93 casos nuevos por cada 100 mil mujeres, estimándose para Vitória/ES una tasa bruta de incidencia de $71,28^{1}$.

El cáncer de mama es una enfermedad que presenta diferentes situaciones de amenaza, generando desánimo psicológico, lo que puede generar ansiedad y un estado depresivo en la mujer. Se observan cambios en su estilo de vida causado por incomodidad física y alteración del concepto de su auto-imagen, llevando a baja estima y disminución de la líbido sexual. También se percibe miedo en cuanto al éxito del tratamiento, así como la posibilidad de su recurrencia y el temor a la muerte. De los tipos de tratamiento, la mastectomía es, sin duda, el procedimiento más traumático de la terapéutica del cáncer de mama ${ }^{2-3}$.

La ansiedad es un síntoma de incidencia bastante común en las pacientes tras el diagnóstico, durante el tratamiento y principalmente en aquellas que precisan someterse a cirugía ${ }^{4-5}$. La ansiedad, a nivel elevado, puede provocar percepciones negativas en cuanto a las habilidades motoras e intelectuales del individuo. Esto, a su vez, interfiere en la atención selectiva y en la codificación de informaciones en la memoria, bloqueando la comprensión y el raciocinio. Varios estudios se realizaron en los últimos años con el objetivo de conocer la relación entre factores emocionales y la vivencia de los pacientes con cáncer de mama ${ }^{6-8}$. 
Estudio observacional de grupo en Londres, Europa, al examinar la ocurrencia de depresión y ansiedad en mujeres en fase inicial de cáncer de mama encontró una prevalencia, en el primer año de la enfermedad, dos veces mayor que la de la población femenina en general ${ }^{6}$.

Estudio con 84 mujeres con cáncer de mama, objetivando evaluar la prevalencia de ansiedad generalizada y depresión mayor, constató que 17,86\% presentaba depresión mayor y $11,9 \%$ ansiedad generalizada ${ }^{7}$.

Estudio reveló una prevalencia expresiva de síntomas clínicamente significativos de ansiedad y depresión entre las pacientes con cáncer de mama. Los síntomas experimentados con más intensidad por las pacientes eran: tensión, miedo inespecífico y preocupaciones difusas ${ }^{8}$.

La ansiedad evaluada en este trabajo se refiere a la ansiedad "natural", como fenómeno de vida cotidiana, con función motivadora, que moviliza recursos para adaptación del individuo a nuevas situaciones. La sensación de ansiedad es una vivencia común, que puede darse en cualquier ser humano y afecta, aproximadamente, a una entre nueve personas ${ }^{9}$.

Evaluar la ansiedad en mujeres mastectomizadas es importante para la identificación de aquellas pacientes con mayor riesgo de desarrollar perturbaciones psicopatológicas en el transcurso del tratamiento. Ante eso, se justifica la realización de este estudio, que tuvo como objetivo evaluar el indicio y el estado de ansiedad en mujeres mastectomizadas y examinar la relación entre el indicio y el estado de esas mujeres, con variables socioeconómicas y clínicas.

\section{METODOLOGÍA}

Se trata de un estudio descriptivo con enfoque cuantitativo, realizado en el Programa de Rehabilitación para Mujeres Mastectomizadas (PREMMA) localizado en el ambulatorio de un hospital que ofrece asistencia de alta complejidad en oncología, en Vitória/Espírito Santo.

En el PREMMA, las mujeres participan de grupos educativos, donde reciben orientaciones referentes a los cuidados con el miembro homolateral en cirugía e informaciones sobre los tratamientos disponibles contra el cáncer de mama. Además de esto, las mujeres cuentan con el apoyo de profesionales de diversas áreas como enfermeros y estudiantes de enfermería, psicóloga, asistente social y fisioterapeuta que las ayudan a luchar con esa nueva realidad vivida. Durante las reuniones del grupo, las mujeres tienen la oportunidad de compartir sobre sus angustias y miedos, de relajarse, participando de dinámicas, y de intercambio de experiencias, lo que es muy importante para ellas, pues así sienten que no están solas en esta lucha contra el cáncer.

La población de la investigación estuvo compuesta por mujeres matriculadas en el PREMMA estando la muestra de estudio constituida por 84 mujeres mastectomizadas. La colecta de datos se realizó por medio de entrevista individual en el período de diciembre de 2009 a marzo de 2010. Como criterios de inclusión se seleccionaron mujeres que tenían más de 21 años, con diagnóstico de cáncer de mama, ausencia de metástasis a distancia, sometidas a intervención quirúrgica, que no tuviesen historia personal de enfermedad psiquiátrica y no fuesen consumidoras 
de drogas ilícitas. Las mujeres solamente participaron del estudio tras el debido esclarecimiento sobre la investigación y su firma del Término de Consentimento Livre e Esclarecido.

Se utilizó una entrevista con registro en formulario para análisis de las variables: edad, grado de instrucción, estado civil, ocupación, renta familiar, apoyo del compañero, ocupación del compañero, apoyo financiero, tipo de cirugía, hospital donde realizó la cirugía, y momento del tratamiento en que llegó al programa de rehabilitación. La variable clase económica fue evaluada a través del formulario de clasificación económica de la Associação Brasileira de Empresas de Pesquisa ${ }^{10}$.

Para evaluar el Indicio y el Estado de Ansiedad se utilizó el instrumento STAI- State Trait Anxiety Inventory, elaborado por Spielberger y colaboradores, conocido en Brasil como Inventario de Ansiedad Indicio (A-indicio) y Estado (A-estado) (IDATE), validado para el portugués por Biaggio y Natalício. Compuesto de dos partes, cada una contiene veinte afirmaciones. El Indicio de Ansiedad evalúa como normalmente la mujer se siente en su vida, medido a través de la escala: Casi Siempre $=4$, Frecuentemente $=3$, Algunas Veces $=2$, Casi Nunca $=1$, y el Estado de Ansiedad evalúa el estado de ansiedad de la mujer en el momento de la entrevista en el PREMMA, medido en la escala: $\mathrm{No}=1$, Un Poco $=2$, Bastante $=3$, Totalmente $=4$. La puntuación de cada instrumento es variable de veinte a ochenta, y el resultado es clasificado de acuerdo con la puntuación. Indican bajo grado de ansiedad los escores de 20 a 40 puntos, medio de 40 a 60 y alto de 60 a 80 puntos $^{3}$.

Para tratamiento estadístico de los datos se utilizó un análisis descriptivo y realizado el test chi-cuadrado para análisis de la relación entre las variables y la ansiedad. Un $p$-valor significante $(p<0,05)$ indica que hay alguna relación entre estas variables. El Paquete Estadístico utilizado fue el SPSS (Social Package Statistical Science) versión 15.0.

Al término del estudio se entregó a las mujeres una carta-respuesta, comunicándoles los resultados del análisis de las escalas del indicio y del estado de ansiedad. En los casos en que se observaron resultados de alto nivel de indicio y/o estado de ansiedad, las mujeres fueron dirigidas al servicio de psicología de la institución.

El proyecto de investigación fue aprobado en el Comitê de Ética em Pesquisa del Centro de Ciências da Saúde da Universidade Federal do Espírito Santo, con número 225/09.

\section{RESULTADOS}

Tras la aplicación y análisis del Inventario de Ansiedad Indicio y Estado (IDATE), se encontró que para el indicio de ansiedad, $79,8 \%$ de las mujeres mastectomizadas tenían un nivel medio de ansiedad, $13,1 \%$ un nivel bajo y $7,1 \%$ un nivel alto de ansiedade (Figura1). 


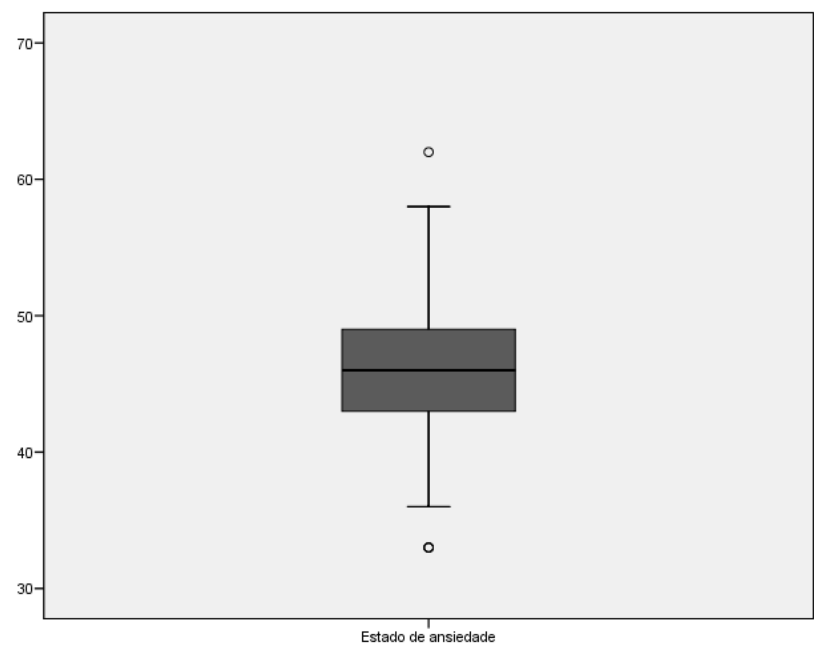

Media $=45,39$

Mediana $=46$

Desvíio $\mathrm{p}=5,2$

Outlier $=33,62$

Figura 1. Estado de ansiedad en mujeres mastectomizadas atendidas en el PREMMA, 2009, Vitória/ES.

En cuanto al estado de ansiedad se observó que 83,3\% tenía un nivel medio de ansiedad, $15,5 \%$ un nivel bajo y $1,2 \%$ un nivel alto. (Figura 2 ).

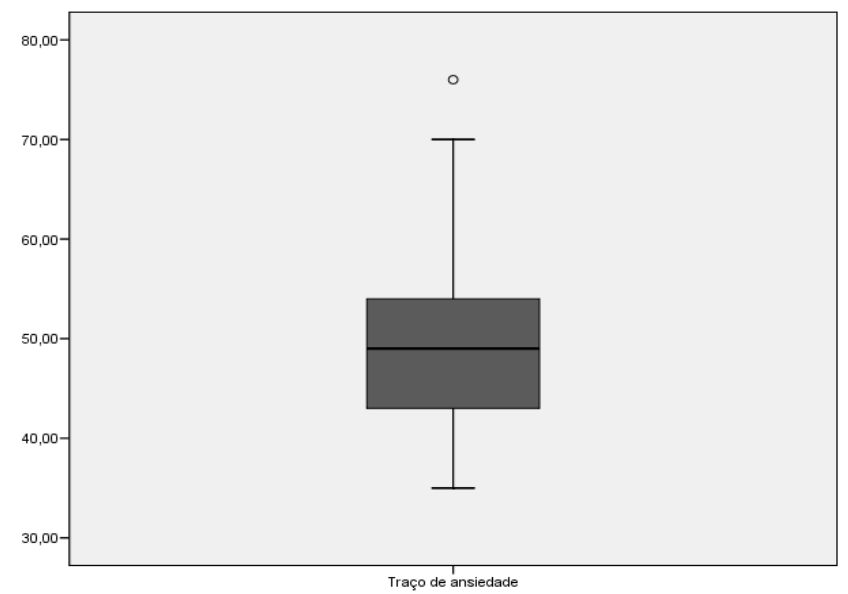

Figura 2. Indicio de ansiedad en mujeres mastectomizadas atendidas en el PREMMA, 2009, Vitória/ES.

En lo que se refiere a las variables socioeconómicas, conforme la Tabla I, se verificó que la edad de las mujeres participantes de la investigación varió de 31 a 60 años, siendo la edad media de 45,5. Se observó también que 39,3\% de estas mujeres tenían edad entre 50 y 59 años, 26,2\% tenían de 40 a 49 años, 26,2\% 60 años o más y $8,3 \%$ tenía de 31 a 39 años.

Respecto al grado de instrución, 44\% de las mujeres poseían enseñanza fundamental incompleta, 16,7\% tenía enseñanza media completa, 13,1\% enseñanza fundamental completa, $10,7 \%$ de las mujeres eran analfabetas, $10,7 \%$ enseñanza media incompleta, y $4,8 \%$ terminabas la enseñanza superior.

Se demostró que de todas las mujeres entrevistadas, 59,5\% están casadas o tienen unión estable, $20,2 \%$ son viudas, $10,7 \%$ son solteras y $9,5 \%$ son separadas 0 divorciadas. 
Sobre la ocupación se verificó que $31 \%$ de las mujeres son amas de casa, $25 \%$ están jubiladas, $19 \%$ ejercen otros tipos de actividad, 8,3\% son servidoras públicas, $8,3 \%$ son profesionales liberales, $7,1 \%$ de las mujeres son empleadas de empresa privada y $1,2 \%$ es agricultora/empleada rural.

De las 84 mujeres entrevistadas, $41,7 \%$ tienen más de uno y hasta tres salarios mínimos, 32,1\% tienen renta familiar de un salario mínimo, 17,9\% tienen más de tres y hasta cinco salarios mínimos, 7,1 \% más de cinco y hasta diez salarios mínimos y $1,2 \%$ tienen renta familiar superior a diez salarios mínimos. De estas mujeres, 53,6\% son de la clase económica $\mathrm{C}, 23,8 \%$ son de la clase económica $B$ y $22,6 \%$ de la clase económica D.

Se verificó que, $74,5 \%$ de las mujeres reciben tanto soporte emocional como financiero de sus compañeros, $11,8 \%$ reciben solo apoyo financiero, 3,9\% reciben apoyo emocional del compañero, y $9,8 \%$ no reciben ayuda alguna de sus compañeros.

En relación al apoyo social se observó que, $75 \%$ de las mujeres no reciben ningún tipo de apoyo, $19 \%$ reciben apoyo de los familiares, 3,6\% de otros, y 2,4 \% reciben apoyo de los amigos.

Tabla I. Características socioeconómicas de las mujeres mastectomizadas atendidas en el PREMMA, 2009, Vitória/ES.

\begin{tabular}{llcc}
\hline \hline & Variables Socioeconómicas & $\mathbf{n}$ & $\mathbf{\%}$ \\
\hline \multirow{4}{*}{ Edad } & 31 a 39 años & 7 & 8,3 \\
& 40 a 49 años & 22 & 26,2 \\
& 50 a 59 años & 33 & 39,3 \\
& 60 años o más & 22 & 26,2 \\
\hline & Analfabeto & 9 & 10,7 \\
& Enseñanza fundamental incompleta & 37 & 44,0 \\
Grado de instrucción & Enseñanza fundamental completa & 11 & 13,1 \\
& Enseñanza medio incompleta & 9 & 10,7 \\
& Enseñanza medio compleao & 14 & 16,7 \\
& Enseñanza superior completa & 4 & 4,8 \\
\hline \multirow{5}{*}{ Estado civil } & Casada / unión estable & 50 & 59,5 \\
& Soltera & 9 & 10,7 \\
& Divorciada / separada & 8 & 9,5 \\
& Viuda & 17 & 20,2 \\
\hline \multirow{5}{*}{ Renta familiar } & Agricultor/ Empleado rural & 1 & 1,2 \\
& Empleado de empresa privada & 6 & 7,1 \\
& Profesional liberal & 7 & 8,3 \\
& Servidor Público & 7 & 8,3 \\
& Ama de casa & 26 & 31,0 \\
& jubilada & 21 & 25,0 \\
& Otras & 16 & 19,0 \\
\hline & Un salario mínimo & 27 & 32,1 \\
& Más de tres hasta cinco salarios & 35 & 41,7 \\
& mínimos & 15 & 17,9 \\
& & &
\end{tabular}




\begin{tabular}{llcc} 
& Más de cinco hasta diez salarios & 6 & 7,1 \\
& mínimos & & \\
& Más de diez hasta quince salarios & 1 & 1,2 \\
& mínimos & 20 & 23,8 \\
& Clase B & 45 & 53,6 \\
Clase econômica & Clase C & 19 & 22,6 \\
\hline \multirow{5}{*}{ Soporte del compañero } & Clase D & 2 & 3,9 \\
& Emocional & 6 & 11,8 \\
& Financiero & 38 & 74,5 \\
& Emocional y financiero & 5 & 9,8 \\
\hline \multirow{5}{*}{ Apoyo social } & Ninguno & 16 & 19,0 \\
& Familiar & 2 & 2,4 \\
& Amigos & 3 & 3,6 \\
& Otros & 63 & 75,0 \\
\hline Total & Ninguno & $\mathbf{8 4}$ & $\mathbf{1 0 0 , 0}$ \\
\hline \hline
\end{tabular}

Entre las variables clínicas, de acuerdo con la Tabla II, en cuanto al local donde las mujeres realizaron cirugía, se encontró que $81 \%$ de las mujeres realizaron cirugía en el Hospital donde ocurrió la investigación, y 19\% en otras instituciones.

En relación al tipo de cirugía, se observó que del total de mujeres participantes de la investigación, 63,1\% tuvieron mastectomía con linfadenectomía, 26,2\% cuadrantectomía con linfadenectomía, 6\% cuadrantectomía sin linfadenectomía, y $4,8 \%$ tuvieron mastectomía sin linfadenectomía.

En cuanto al momento en que las mujeres llegaron al PREMMA, se observó que, $54,8 \%$ llegó después de la realización de la cirugía, 15,5\% después de Quimioterapia/Radioterapia/Hormonioterapia, 11,9\% realizando radioterapia, 9,5\% realizando la quimioterapia, 4,8\% en tratamiento de hormonioterapia, y $3,6 \%$ tras el diagnóstico, sin tratamiento.

Tabela II. Características clínicas de las mujeres mastectomizadas atendidas en el PREMMA, 2009, Vitória/ES.

\begin{tabular}{llcc}
\hline \hline & Variables Clínicas & n & \% \\
\hline Tuvo cirugía en el & Sí & 68 & 81,0 \\
Hospital de estudio & No & 16 & 19,0 \\
\hline & Cuadrantectomia sin linfadenectomía & 5 & 6,0 \\
& $\begin{array}{l}\text { Cuadrantectomía con } \\
\text { Tipo de cirugía }\end{array}$ & 22 & 26,2 \\
& Mastectomía sin linfadenectomía & 4 & 4,8 \\
& Mastectomía con linfadenectomia & 53 & 63,1 \\
\hline
\end{tabular}




\begin{tabular}{llcc}
\hline & Pos diagnóstico - sin tratamiento & 3 & 3,6 \\
Momento de la & Pos cirugía & 46 & 54,8 \\
matrícula en el & En quimioterapia & 8 & 9,5 \\
PREMMA & En radioterapia & 10 & 11,9 \\
& En hormonioterapia & 4 & 4,8 \\
& Pos quimioterapia/radioterapia & 13 & 15,5 \\
\hline Total & /hormonioterapia & $\mathbf{8 4}$ & $\mathbf{1 0 0 , 0}$ \\
\hline \hline
\end{tabular}

Al relacionar el indicio de ansiedad con las variables socioeconómicas y clínicas no hubo relación significativa, es decir, $(p>0,05)$. Sin embargo, al relacionar el estado de ansiedad y las variables socioeconómicas y clínicas, se observó solamente una relación significativa, $(p=0,004)$, con el momento en que la mujer se matriculó en el PREMMA.

\section{DISCUSIÓN DE LOS RESULTADOS}

Con relación al indicio y estado de ansiedad en mujeres con cáncer de mama, investigaciones demuestran que las mujeres reaccionan con ansiedad en diversas fases. En el momento inmediato tras el diagnóstico de cáncer de mama, las mujeres presentan alto nivel de ansiedad ${ }^{3}$. Sin embargo, en el periodo pre y poós-operatorio elas presentan indicio y estado de media ansiedad ${ }^{4,11-12}$. Mujeres mastectomizadas que toman tamoxifeno también tienen nivel medio de indicio y estado de ansiedad ${ }^{5}$. Estos hallazgos corroboran lo observado en el presente estudio, que encontró nivel medio para el indicio y el estado de ansiedad de las mujeres mastectomizadas.

En cuanto a la edad, los datos del estudio presentan el mayor percentual $(65,5 \%)$ de mujeres en la franja de edad de 40 a 59 años, estando en consonancia con los hallazgos de diversas investigaciones ${ }^{1,4-6,12-14}$.

De la misma forma, la varible grado de instrucción demostró que la mayor parte de las mujeres mastectomizadas participantes de ese estudio, tienen enseñanza fundamental incompleta, semejante a otros estudios realizados ${ }^{13-15}$.

De las participantes del presente estudio, hubo prevalencia de mujeres casadas, que tenían renta mensual de hasta 3 salarios mínimos y pertencían a la clase económica C. Resultados similares se encontraron al estudiar la depresión y la ansiedad en pacientes con cáncer de mama, donde la mayoría de las mujeres estaba casada ye tenía renta familiar total de hasta tres salarios mínimos ${ }^{7}$.

Retornando a la cuestión del estado civil, la prevalencia de casadas también fue observada en otros estudios desarrollados con mujeres mastectomizadas en el Espírito Santo ${ }^{4-5,12-15}$.

Al analizar el perfil de las mujeres en tratamiento de cáncer de mama en la ciudad de Maringá/PR, investigación verificó que el 32\% de las mujeres pertenecía a la clase económica $\mathrm{C}$, lo que está de acuerdo con el presente estudio ${ }^{16}$. Aunque estudio en Vitória/ES con 270 mujeres mastectomizadas en tratamiento con tamoxifeno encontró que $47,8 \%$ de las mujeres pertenecían a la clase económica $D$ y $20 \%$ a la clase $E^{15}$. 
Respecto a la ocupación, se encontró en este estudio, que $31 \%$ son amas de casa, corroborando con este dato la investigación que verificó que la mayoría de las mujeres $64 \%$ era ama de casa y solo $14 \%$ eran jubiladas ${ }^{16}$.

Se observó que la mayoría de las mujeres con compañero, recibe tanto ayuda emocional como financiera de sus compañeros, concordando con los hallazgos de otros trabajos $^{17-18}$

La presencia del compañero es altamente significativa, en lo que se refiere a la creación de un ambiente saludable para que la mujer pueda sentirse nuevamente integrada en el contexto familiar. Es también muy importante que el compañero se muestre dispuesto a ofrecer afecto, así la paciente se sentirá acogida y comprendida por él ${ }^{2}$.

La mayoría de las mujeres realizó cirugía en el Hospital en que ocurrió la investigación. Esto se debe, probablemente, a que el lugar de realización de esta investigación fue el PREMMA, que está vinculado a este hospital. Al recibir el diagnóstico de cancer de mama, las mujeres fueron dirigidas a matricularse en el Programa de Rehabilitación, siguiendo el flujo de atención de la institución ${ }^{12}$, lo que, posiblemente, no sucede cuando el tratamiento es realizado en otras instituciones hospitalarias.

De las 84 mujeres investigadas, 63,1\% realizaron mastectomía com linfadenectomía. Estudio realizado en 2004 sobre el perfil de las mujeres matriculadas en el PREMMA verificó que $38,3 \%$ había realizado cuadrantectomía y $57,4 \%$ mastectomía ${ }^{19}$.

Esa prevalencia de mastectomía com linfadenectomía, tal vez está justificada, por el hecho de que esta investigación ocurrió dentro del PREMMA que tiene como sujeto principal de su actuación, a las mujeres mastectomizadas, además, estas mujeres permanecen más tiempo participando del programa para prevención de las complicaciones pos-mastectomía y linfadenectomía 4 .

Por otro lado, podemos pensar en la hipótesis de que esas mujeres llegaran para diagnóstico con estadio avanzado de la enfermedad. Buscando averiguar esa posibilidad, encontramos que investigación realizada entre mujeres mastectomizadas en tratamiento de tamoxifeno en Vitoria/ES encontró que 41,82\% estaban en estadio II y $34,55 \%$ en estadio III $^{15}$. Estudio descriptivo de 2930 registros de casos analíticos de tumores malignos en la mama atendidos de enero de 2000 a diciembre de 2006 en esa misma institución hospitalaria de Vitória/ES verificó que 48,1\% de los casos eran de estadio precoz (hasta IIB), 23,2\% de estadio tardío (III y IV) y en $28,7 \%$ de las fichas de registro de tumor, la varible estadio no estaba rellenada ${ }^{13}$.

En relación al momento en que las mujeres de la investigación se matricularon en el PREMMA, 54,8\% fueron admitidas tras el tratamiento cirúrgico.

En este estudio, se encontró significancia estadística en el cruce del estado de ansiedad con el momento en que la mulher fue matriculada en el PREMMA, $p=0,004$, o sea, hay relación entre estas variables. Corroborando con este dato, estudio realizado para evaluar los efectos de la implantación del PREMMA como intervención de enfermería en el pre-operatorio de mujeres con diagnóstico de cäncer de mama, se mostró se que las mujeres que fueron matriculadas en el PREMMA antes de la cirugía presentaron bajo nível de ansiedad comparado con aquellas no matriculadas 
antes de la cirugía. A pesar de que el Programa había sido creado con el objetivo de realizar todas las intervenciones en el pos-operatorio, los resultados de ese estudio fueron muy importantes, e influyeron en la reorganización del flujograma de atención y admisión precoz de las mujeres en el Programa ${ }^{12}$.

En cuanto a las demás variables socioeconómicas y clínicas no hubo significancia estadística con el traço y estado de ansiedad. Confirmando estos achados, estudio en pacientes con cáncer de mama, no encontró relación significativa entre las variables demográficas y el diagnóstico de ansiedad ${ }^{7}$.

\section{CONCLUSIÓN}

Los resultados de este estudio mostraron que la mujer en la vivencia del cáncer de mamaapresenta un nivel medio en relación al traço y al estado de ansiedad, siendo una situación esperada y corrobora los resultados de otros estudios presentes en la literatura. Sin embargo, el hecho de que esta mujer mastectomizada esté vinculada a un programa de rehabilitación, que trabaja de manera interdisciplinar y le ofrece la oportunidad de expresar sus sentimientos, angustias y dudas tal vez justifique que los escores no presentaran niveles más altos de ansiedad, teniendo en cuenta el momento desafiador que ella afronta.

También encontramos como resultado relevante para la reducción de la ansiedad la inserción más precoz de la mujer en el programa, considerando que la atención interdisciplinar que entre otras cosas ofrece soporte emocional y orienta a la mujer y a toda su familia necesita ser implantado cada vez más precozmente en el tratamiento del cáncer de mama. Así pues, se percibe que algunas mujeres $(3,6 \%)$ ya están siendo dirigidas al Programa precozmente y que la participación en el programa después del diagnóstico y antes de realizar cualquier tratamiento provoca un impacto en su vida, disminuyendo el estado de ansiedad de estas mujeres mastectomizadas.

Son necesarias nuevas investigaciones, pero los resultados señalan que los profesionales de salud precisan estar atentos a los aspectos emocionales de la mujer, para que esta pueda enfrentar el cáncer con disposición y vigor, libre de mayores trastornos psicopatológicos, recibiendo durante todo el tratamiento una asistencia de calidad.

\section{REFERENCIAS}

1. Brasil. Ministério da saúde. Secretaria de Atenção à saúde. Instituto Nacional do Câncer. Coordenação de prevenção e vigilância de câncer. Estimativas 2012: incidência de câncer no Brasil. Rio de Janeiro(RJ): Inca, 2011. Disponível em: $\leq$ http://www.inca.gov.br/conteudo >.

2. Vieira CP, Lopes MHBM, Shimo AKK. Sentimentos e experiências na vida das mulheres com câncer de mama. Rev Esc Enferm USP. 2007; 41(2): 311-6.

3. Amorim MHC. A enfermagem e a psiconeuroimunologia no câncer de mama [tese]. Rio de Janeiro: Universidade Federal do Rio de Janeiro - Escola de Enfermagem Ana Nery; 1999

4. Repossi C. Os efeitos da intervenção de enfermagem-relaxamento no sistema imunológico de mulheres com diagnóstico de câncer de mama [Dissertação]. Vitória: Universidade Federal do Espírito Santo - Mestrado em Saúde Coletiva; 2008. 
5. Olympio PCAP. Níveis de imunoglobulina A salivar, ansiedade, estresse e depressão de mulheres mastectomizadas em uso de tamoxifeno [Dissertação]. Vitória: Universidade Federal do Espírito Santo - Mestrado em Saúde Coletiva; 2008.

6. Burguess C, Cornelius V, Love S, Graham J, Richards M, Ramirez A. Depression and anxiety in women with early breast cancer: five year observational cohort study. BMJ. 2005; 330(7493): 702-705.

7. Souza FGM, Ribeiro RA, Silva MSB, Ivo PSA, Lima Jr. VS. Depressão e ansiedade em pacientes com câncer de mama. Rev de Psiquiatria Clínica. 2000; 27(4): 207-214.

8. Pelegrini LG, Cerqueira JA, Peres RS. Morbidade psicológica em mulheres mastectomizadas: influências das reações emocionais ao câncer de mama. In: XII Seminário de Iniciação Científica, 2008 Out 8-9; Uberlândia.

9. Kaplan HI. Sadock Bj, Grebb JA. Compêndio de psiquiatria: ciência do comportamento e psiquiatria clinica. Porto Alegre: Artmed; 2003.

10. ABEP - Associação Brasileira de Empresas de Pesquisa. Critério de Classificação Econômica Brasil, 2010. Disponível em: <http://www.abep.org>.

11. Medeiros RHA, Nunes MLT. A influência do vídeo de informação adicional em pacientes submetidas a mastectomia: o estudo da ansiedade. Psicologia em Estudo. 2001; 6(2): 95-100.

12. Caniçali RA, Figueiredo GB. A inserção de mulheres com diagnóstico de câncer de mama em um programa de reabilitação [trabalho de conclusão de curso]. Vitória: Universidade Federal do Espírito Santo, Curso de Enfermagem, Departamento de Enfermagem; 2006.

13. Silva PF. Perfil de mulheres com câncer de mama atendidas em Vitória-ES: influências das variáveis sociodemográficas com o estadiamento clínico do tumor antes do tratamento [Dissertação]. Vitória: Universidade Federal do Espírito Santo Mestrado em Saúde Coletiva; 2009.

14. Mai Junior AAM, Pereira, JR. Depressão em mulheres mastectomizadas [trabalho de conclusão de curso]. Vitória: Universidade Federal do Espírito Santo, Curso de Enfermagem, Departamento de Enfermagem; 2009.

15. Leite FMC, Bubach S, Amorim MHC, Castro DS, Primo CC. Mulheres com diagnóstico de câncer de mama em tratamento com tamoxifeno: perfil sociodemográfico e clínico. Revista Brasileira de Cancerologia 2011; 57(1):15-21.

16. Campana HCR. Perfil de mulheres com câncer de mama [Dissertação]. Maringá: Universidade Estadual de Maringá - Mestrado em enfermagem; 2007.

17. Sales CACC, Scandiuzz LPD, Anjos, ACY. Qualidade de vida de mulheres tratadas de câncer de mama: Funcionamento Social. Rev Bras de Cancerologia. 2001; 47(3): 263-272.

18. Seidl EMF, Troccoli BT, Zannon CMLC. Pessoas vivendo com HIV/AIDS: enfrentamento, suporte social e qualidade de vida. Psicologia Reflexão e Crítica. 2005; 18(2):188-95.

19. Boldrini R, França T. Perfil de mulheres mastectomizadas de um programa de reabilitação em Vitória/ES [trabalho de conclusão de curso]. Vitória: Universidade Federal do Espírito Santo, Curso de Enfermagem, Departamento de Enfermagem; 2004.

SSN 1695-6141

() COPYRIGHT Servicio de Publicaciones - Universidad de Murcia 